\title{
Wavefront degradation of a 200 TW laser from heat-induced deformation of in-vacuum compressor gratings
}

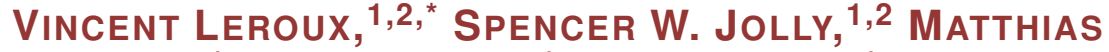 \\ Schnepp, ${ }^{1}$ Timo Eichner, ${ }^{1}$ SÖREn Jalas, ${ }^{1}$ MANUEl KIRChen, ${ }^{1}$ \\ Philipp Messner, ${ }^{1}$ Christian Werle, ${ }^{1}$ Paul Winkler, ${ }^{1,3}$ And \\ ANDREAS R. MAIER ${ }^{1}$ \\ ${ }^{1}$ Center for Free-Electron Laser Science \& Department of Physics, Universität Hamburg, Hamburg, \\ Germany \\ ${ }^{2}$ Institute of Physics of the ASCR, ELI-Beamlines project, Prague, Czech Republic \\ ${ }^{3}$ Deutsches Elektronen-Synchrotron DESY, Hamburg, Germany \\ *vincent.leroux@desy.de
}

\begin{abstract}
High-repetition-rate high-power laser systems induce a high average power heat deposition into the gold-coated diffraction gratings. To study the effects of the thermal expansion of in-vacuum Pyrex gratings on the laser properties, we scan the pulse energy and repetition rate of a 200 TW laser system while monitoring the laser wavefront. Through the measured changes in laser divergence and focusability, we define an average power limit below which the in-vacuum compressor can be used with no degradation of the laser focus quality.
\end{abstract}

(C) 2018 Optical Society of America under the terms of the OSA Open Access Publishing Agreement

OCIS codes: (230.1950) Diffraction gratings; (140.6810) Thermal effects; (120.5050) Phase measurement; (320.7090) Ultrafast lasers.

\section{References and links}

1. C. Danson, D. Hillier, N. Hopps, and D. Neely, "Petawatt class lasers worldwide," High Power Laser Sci. Eng. 3, e3 (2015).

2. G. A. Mourou, T. Tajima, and S. V. Bulanov, “Optics in the relativistic regime," Rev. Mod. Phys. 78, 309-371 (2006).

3. J. Hein, S. Podleska, M. Siebold, M. Hellwing, R. Bödefeld, R. Sauerbrey, D. Ehrt, and W. Wintzer, "Diode-pumped chirped pulse amplification to the joule level," Appl. Phys. B 79, 419-422 (2004).

4. J. Tümmler, R. Jung, H. Stiel, P. V. Nickles, and W. Sandner, "High-repetition-rate chirped-pulse-amplification thin-disk laser system with joule-level pulse energy," Opt. Lett. 34, 1378-1380 (2009).

5. C. W. Siders and C. Haefner, "High-power lasers for science and society," Tech. rep., Lawrence Livermore National Lab. (2016).

6. N. Bonod and J. Neauport, "Diffraction gratings: from principles to applications in high-intensity lasers," Adv. Opt. Photonics 8, 156-199 (2016).

7. O. Loebich, "The optical properties of gold," Gold Bull. 5, 2-10 (1972).

8. Z. Li, K. Tsubakimoto, H. Yoshida, Y. Nakata, and N. Miyanaga, "Degradation of femtosecond petawatt laser beams: Spatio-temporal/spectral coupling induced by wavefront errors of compression gratings," Appl. Phys. Express 10, 102702 (2017).

9. G. Genoud, M. S. Bloom, J. Vieira, M. Burza, Z. Najmudin, A. Persson, L. O. Silva, K. Svensson, C.-G. Wahlström, and S. P. D. Mangles, "Increasing energy coupling into plasma waves by tailoring the laser radial focal spot distribution in a laser wakefield accelerator," Phys. Plasmas 20, 064501 (2013).

10. S. Backus, R. Bartels, S. Thompson, R. Dollinger, H. C. Kapteyn, and M. M. Murnane, "High-efficiency, single-stage 7-khz high-average-power ultrafast laser system," Opt. Lett. 26, 465-467 (2001).

11. S. Fourmaux, C. Serbanescu, L. Lecherbourg, S. Payeur, F. Martin, and J. C. Kieffer, "Investigation of the thermally induced laser beam distortion associated with vacuum compressor gratings in high energy and high average power femtosecond laser systems," Opt. Express 17, 178-184 (2009).

12. D. A. Alessi, P. A. Rosso, H. T. Nguyen, M. D. Aasen, J. A. Britten, and C. Haefner, "Active cooling of pulse compression diffraction gratings for high energy, high average power ultrafast lasers," Opt. Express 24, 30015-30023 (2016).

13. N. Delbos, C. Werle, I. Dornmair, T. Eichner, L. Hübner, S. Jalas, S. Jolly, M. Kirchen, V. Leroux, P. Messner, M. Schnepp, M. Trunk, P. Walker, P. Winkler, and A. Maier, "LUX: A laser-plasma driven undulator beamline," Nucl. Instruments Methods Phys. Res. Sect. A: Accel. Spectrometers, Detect. Assoc. Equip. (2018). 
14. J.-C. Chanteloup, "Multiple-wave lateral shearing interferometry for wave-front sensing," Appl. Opt. 44, 1559-1571 (2005).

15. U. Hahn and K. Zapfe, "Guidelines for UHV-components at DESY," Tech. rep., Deutsches Elektronen-Synchrotron (2010).

16. S. Akturk, X. Gu, P. Bowlan, and R. Trebino, "Spatio-temporal couplings in ultrashort laser pulses," J. Opt. 12, 093001 (2010).

\section{Introduction}

Since the development of the ultrafast laser technology, an increasing number of laboratories have access to several hundreds of terawatt and up to a few petawatts of laser power [1]. Such beams can be focused down to reach intensities well above $10^{18} \mathrm{~W} / \mathrm{cm}^{2}$, which corresponds to the regime where electrons accelerated by the laser electric field can reach relativistic energies [2]. In parallel to the increase of peak power, constant effort has been undertaken to increase the repetition rate, for example by using diode-based pump lasers [3] or thin disks as amplifier medium instead of rods [4]. The increase of repetition rate allows laboratories to move from single-shot proof-of-principle experiments to systematic studies and parameter scans backed up by statistical data, which enables a deeper and more consistent analysis. Furthermore, with the development of high-power laser systems as drivers for secondary sources such as laser-plasma accelerators for instance, increasing the repetition rate is necessary to obtain performances required for applications. Additionally, increasing the repetition rate of laser systems allows for active feedback and therefore a better control of the pulse properties [5].

Nowadays, several hundreds of terawatt laser systems with a few hertz repetition rate are commercially available. Such lasers are typically based on titanium-sapphire technology, with broadband pulses that can be compressed to below $50 \mathrm{fs}$. Even though recent progress in multilayer dielectric gratings has been made to improve the diffracted bandwidth [6], most of the compressors feature gold coated gratings as they are suitable for broadband lasers. Gold, however, absorbs $3 \%$ to $5 \%$ of the incident energy [7], which combined with the high repetition rate leads to a high average power absorbed by the grating substrate. This induces a thermal load that can only be extracted via radiative cooling due to the necessity of operating high power compressor in a vacuum environment with limited contacts to the optomechanics to reduce mechanical stresses. The absorbed energy therefore stays within the substrate as heat and deforms the grating surface. Thus, the reflected laser wavefront will be deformed. Moreover, as the beam is spatially dispersed between the gratings, the changes of the wavefront can couple into the temporal properties of the pulse and be detrimental to the overall compression [8]. Eventually, the heat can lead to catastrophic damage of the optic, e.g. if the temperature is higher than the melting point of the gold coating. The deformation of the laser wavefront itself has a direct negative impact on the focusability of the beam, which heavily decreases the available peak intensity as well as the energy contained within the central part of the focal spot. This fraction of the pulse energy is typically a crucial parameter which determines the efficiency of a process [9].

Previous work has reported this issue at different pulse energies [10-12] and proposed solutions to mitigate or compensate the thermal expansion. Fourmaux et al. [11] propose to adjust the position of the off-axis parabola to compensate the defocusing of the $110 \mathrm{~mJ} 100 \mathrm{~Hz}$ laser used. On the contrary, Alessi et al. [12] tackle the source of the problem by actively cooling the grating substrate to efficiently extract heat and therefore reduce the surface deformation for a PW-class grating compressor, measured using a diode array with equivalent average power. Here, we report, to our knowledge, the first direct measurement of heat-induced wavefront distortion in gold-coated gratings of a $100 \mathrm{TW}$ class vacuum compressor using the actual high-energy ultrashort laser to both heat up the gratings and diagnose their deformations. The observed deformations could therefore be used to predict the grating behavior during an experiment without relying on assumptions on the influence of vacuum, beam size or pulse duration for instance. We 
first describe the experimental setup as well as the laser parameters used for this purpose. Then, we present the results of the laser energy and repetition rate scans on the output laser divergence, which directly relates to the focus position. Finally, we emphasize on the focus quality itself through the observation of the overall wavefront transient deformation.

\section{Experimental setup}

The ANGUS 200 TW laser system is a double CPA Ti:sapphire system mainly used to drive the laser-driven plasma accelerator called LUX [13]. It can deliver up to $5 \mathrm{~J}$ on target at a maximum repetition rate of $5 \mathrm{~Hz}$. The compressor is composed of two gold-coated holographic gratings with a Pyrex substrate operated in double pass, an incidence angle of $51.4^{\circ}$, and 1480 lines $/ \mathrm{mm}$. The first grating dimensions are $210 \mathrm{~mm}$ wide and $200 \mathrm{~mm}$ high, and the second grating dimensions are $300 \mathrm{~mm}$ wide and $200 \mathrm{~mm}$ high. Although the laser bandwidth allows a compression down to $25 \mathrm{fs}$, we relaxed this parameter and use a more reproducible pulse length of $40 \mathrm{fs}$ for this measurement, as in everyday operation. The near field intensity distribution shows a super-Gaussian-like profile with a full width at half maximum of $70 \mathrm{~mm}$. To measure the heat induced wavefront deformations, we scanned the energy from $0.6 \mathrm{~J}$ to $6.0 \mathrm{~J}$ at the compressor entrance using a half-waveplate and thin film polarizer based attenuator. This corresponds to an average fluence on the first grating surface ranging from $10 \mathrm{~mJ} / \mathrm{cm}^{2}$ to $100 \mathrm{~mJ} / \mathrm{cm}^{2}$. The laser was run for a duration of 30 minutes for the low energy steps, and up to 90 minutes for the high energy steps. Additionally, after selected tests, the laser energy was tuned down to $150 \mathrm{~mJ}$ for 20 minutes to measure the cooling behavior of the gratings while still being able to measure the laser parameters. The repetition rate was scanned by changing the laser internal trigger to allow only every other or every third pulse to be sent to the multipass power amplifiers. We therefore scanned the repetition rates of $0.5 \mathrm{~Hz}, 1.0 \mathrm{~Hz}, 1.67 \mathrm{~Hz}, 2.5 \mathrm{~Hz}$, and $5.0 \mathrm{~Hz}$.

To monitor the laser parameters, the leakthroughs from two mirrors are used before and after the compressor, as shown in Fig. 1. These leakthroughs are then attenuated, demagnified, and split towards different diagnostics: the pulse energy, spectrum, and near field and far field distributions are measured before and after the compressor, and recorded for every shot in the control system with a unique shot number. The wavefront is measured after the compressor using a four-wave lateral shearing interferometer wavefront sensor [14] which has been used to optimize the wavefront before the compressor prior to the experiment with a mechanical deformable mirror. The post-compressor diagnostics have inherent astigmatism, therefore the wavefront measurements presented in the following sections show relative results. This has the advantage of ignoring the aberrations of the setup and the laser itself, and only focus on the transient deformations of the optics. Using these diagnostics, we can ensure that the laser beam does not change before the compressor and thus rely on the post-compressor diagnostics. All the data reported here was acquired for every individual shot. Additionally, an infrared camera with a spectral range of $7.5 \mu \mathrm{m}$ to $14 \mu \mathrm{m}$ was used to monitor the first grating temperature from outside of the compressor through a $\mathrm{ZnSe}$ window. As the emissivity of the gold coating is low $\left(\varepsilon_{\text {gold }}<0.05\right)$ [7], only the heat emitted from the side of the grating substrate was visible and measurable $\left(\varepsilon_{\text {glass }}>0.8\right)$. It is however a good indicator of the dynamics of the temperature increase within the grating.

As the full energy of the ANGUS laser was used, the compressor was operated at a pressure below $10^{-6} \mathrm{mbar}$, the quality of the vacuum was continuously monitored by a residual gas analyzer to ensure that no contamination could disturb the experiment by increasing the absorption or lowering the damage threshold of the gratings. We defined the good quality of the vacuum by measuring that the partial pressure of masses above $45 \mathrm{amu}$ is less than $10^{-3}$ of the total pressure [15]. The data was saved and archived into the control system and post-analysis revealed that the vacuum environment did not change during the experiment. The full power laser beam was then sent through the transport beamline towards the experimental area to be dumped, as 
only a fraction is sampled for the post-compressor diagnostics. Therefore, the parameter scans reported here are done in realistic conditions: the vacuum system, the laser beam size, energy, and pulse duration are similar to those used for the LUX experiment [13] and thus reflect the actual behavior of the grating compressor during everyday operation. While the data reported is specific to the ANGUS laser system and compressor, it is a rather standard design concerning the grating substrate, the filling ratio or the fluence on the optics. We therefore believe that the conclusions can be, to some extent, adapted to different designs and laser parameters.

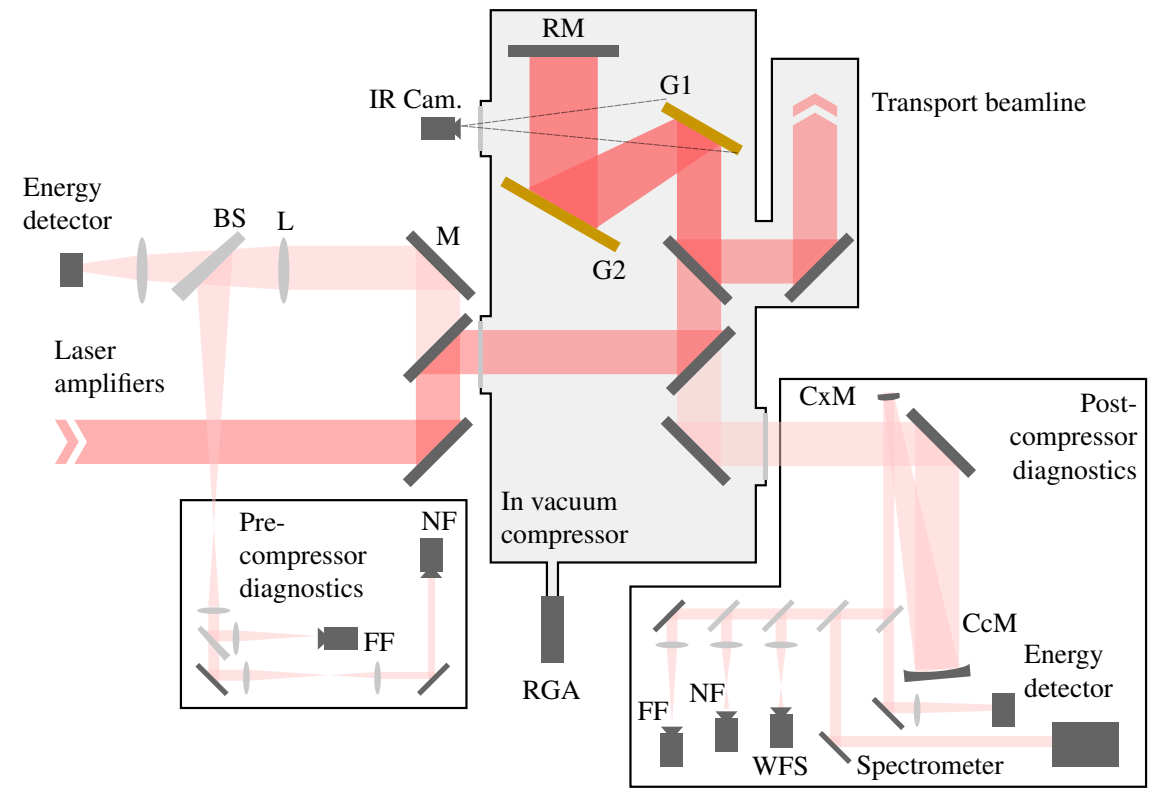

Fig. 1. Schematic of the ANGUS pre- and post-compressor diagnostics. NF, near field camera; FF, far field camera; IR Cam., infrared camera; WFS, wavefront sensor; M, mirror; L, lens; BS, beam splitter; G, grating; RM, roof mirror; CcM, concave mirror; CxM, convex mirror; RGA, residual gas analyzer.

\section{Effects on divergence}

We first investigate the change of the beam divergence as it directly relates to a shift of the longitudinal focus position. The impact of the input beam fluence in the grating plane is studied at a fixed repetition rate of $5 \mathrm{~Hz}$, and then the repetition rate is varied while the fluence is set to a fixed value of $50 \mathrm{~mJ} / \mathrm{cm}^{2}$.

\subsection{Energy scan}

Figure 2 shows the relative change of the beam divergence over time in the horizontal (top) and vertical plane (bottom) for five fluence steps measured with the wavefront sensor, which correspond to input energies of $0.6 \mathrm{~J}, 1.8 \mathrm{~J}, 3.0 \mathrm{~J}, 3.6 \mathrm{~J}$, and $4.2 \mathrm{~J}$. A clear positive correlation between the input fluence and the increase of divergence can be observed. As expected, as more energy is absorbed by the substrate, the higher thermal load leads to a higher deformation of the grating surface. The second observation is that the horizontal divergence increases at a much higher rate than the vertical divergence, leading to an increase of astigmatism of the beam. Furthermore, the horizontal divergence seems to keep increasing linearly after an exponential growth while the vertical divergence saturates or even decreases after a similar exponential behavior. 


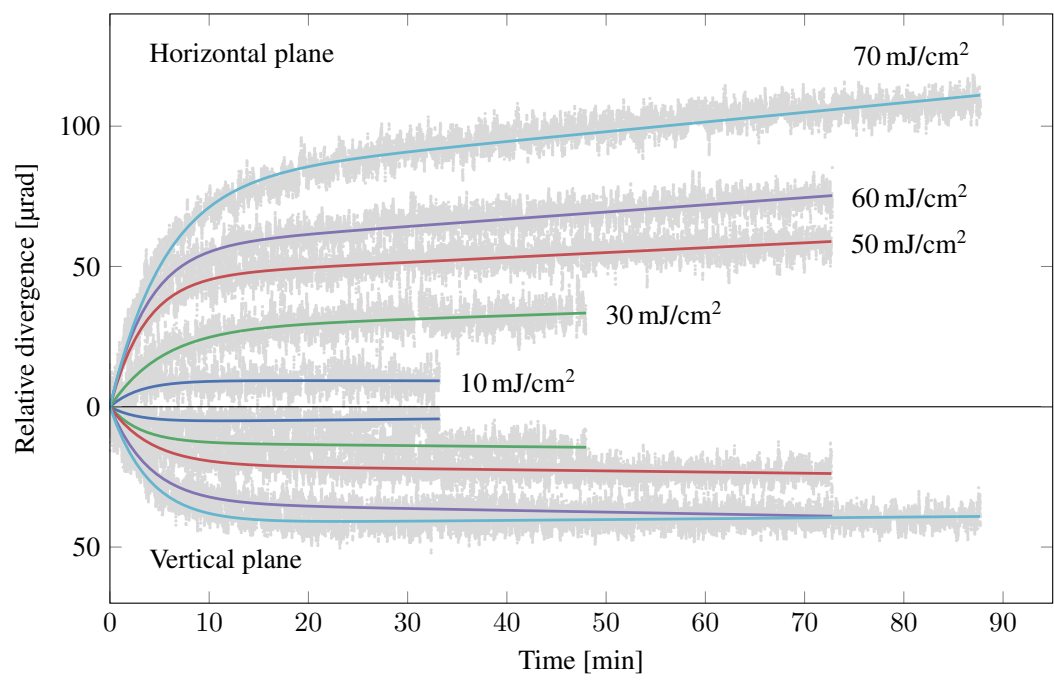

Fig. 2. Evolution of the laser beam horizontal (top) and vertical (bottom) divergence measured after the grating compressor for different fluence level from $10 \mathrm{~mJ} / \mathrm{cm}^{2}$ to $70 \mathrm{~mJ} / \mathrm{cm}^{2}$ at a repetition rate of $5 \mathrm{~Hz}$. The single-shot data (gray dots) is overlaid by a fit (colored solid line) in a good agreement with the measurements.
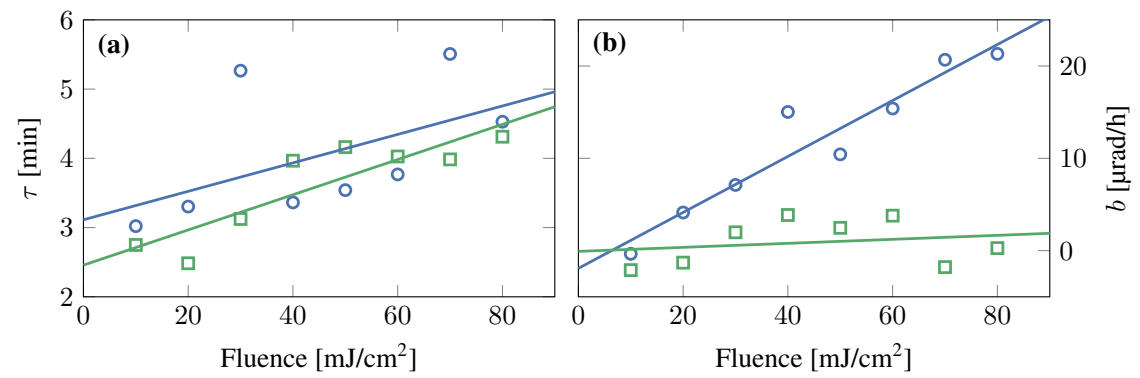

Fig. 3. Exponential constant (a) and linear growth rate (b) of the fitted curves (solid lines on Fig. 2) for the different measured input fluences in the horizontal (blue circles) and vertical (green squares) planes. A linear fit (solid line) highlights the behavior of the scan.

The temporal evolution of the beam divergence is fitted with the sum of an exponential increase and a linear curve $f(t)=a(1-\exp (-t / \tau))+b t$, based on the observed pattern. All fits show a very good agreement with the measured data and we could then retrieve two time constants: the exponential time constant $\tau$ and a linear growth rate $b$ which are plotted in Fig. 3. While the exponential time constant increases similarly in both planes with the input fluence, the linear growth rate is only significant in the horizontal plane. The horizontal divergence does not show saturation for higher input fluences. In an attempt to reach such a regime, an additional 4 hour run was measured with an input energy of $6 \mathrm{~J}$ at $5 \mathrm{~Hz}$. After the exponential growth happening in the first 10 to 20 minutes, the horizontal divergence increases quasi-linearly for two hours before saturating around $190 \mu \mathrm{rad}$. In the last two hours of the run, it then decreased with a rate on the order of $10 \mu \mathrm{rad} / \mathrm{h}$. The vertical divergence, on the other hand, was linearly increasing with a rate of $3 \mu \mathrm{rad} / \mathrm{h}$ after the exponential growth. To describe this asymmetric behavior more quantitatively, further studies and simulations of both the thermal expansion of the substrate and of the affected laser pulse spatial and spectral properties are necessary. 
One can also notice on Fig. 2 that the increase in divergence between two different energy levels is not constant. This can be explained by a drawback in our experimental procedure. Due to the amount of laser time required, the data sets presented here were acquired over the course of several days. Therefore, the time between two runs has not always been long enough to allow the gratings temperature to go back to the initial temperature of $20^{\circ} \mathrm{C}$. Thus, the exponential curve on Fig. 2 would start with a higher offset which is not visible in these results showing only the relative changes during a run.

Finally, we report that for the three highest input fluence levels, we observed a deterioration of the first grating coating, which most likely increased the absorbed laser energy by the grating. Thus, the results at a repetition rate lower than $5 \mathrm{~Hz}$ or an input fluence higher than $80 \mathrm{~mJ} / \mathrm{cm}^{2}$ have been measured on the deteriorated coating. While it affects the amplitude of the wavefront deformations and the time constants, the overall trends reported would stay valid as long as the coating quality stays identical for a given set of measurements.

\subsection{Repetition rate scan and average power dependency}

The repetition rate has a direct influence on the divergence evolution as can be seen on Fig. 4 , where it is varied from $1 \mathrm{~Hz}$ to $5 \mathrm{~Hz}$ at a constant input fluence of $50 \mathrm{~mJ} / \mathrm{cm}^{2}$.

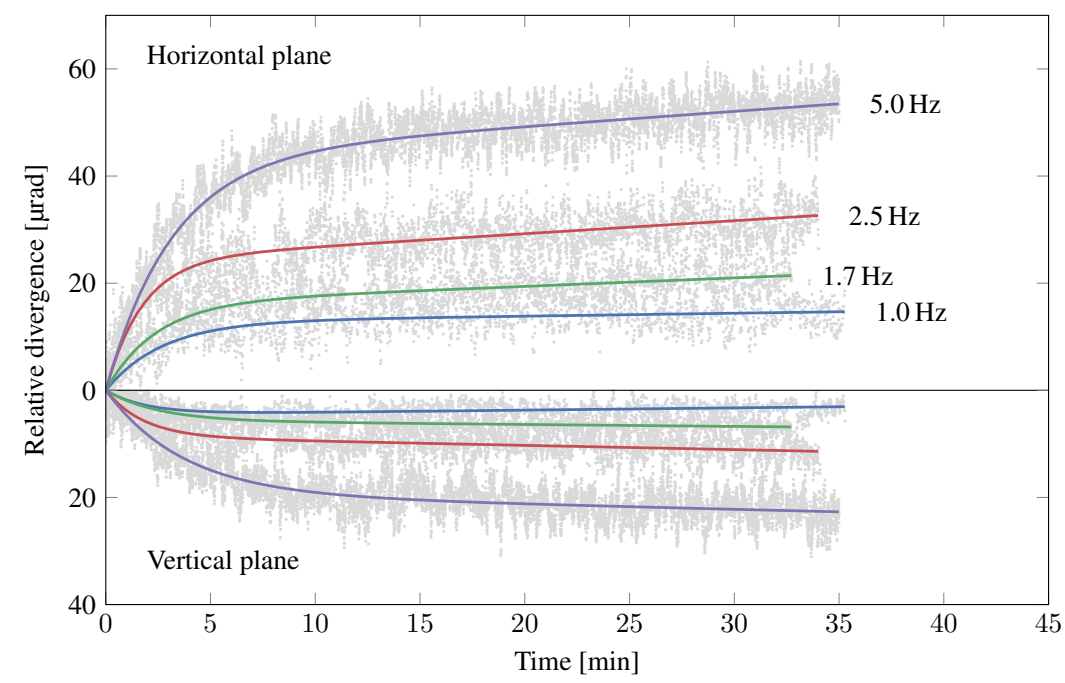

Fig. 4. Evolution of the laser beam horizontal (top) and vertical (bottom) divergence measured after the grating compressor for different repetition rates from $1 \mathrm{~Hz}$ to $5 \mathrm{~Hz}$ at a fluence of $50 \mathrm{~mJ} / \mathrm{cm}^{2}$. The data (gray dots) is again overlaid by a fit (colored solid line).

As the repetition rate increases at a constant input energy, the average power and thus the heat absorbed by the gratings increases as well. Therefore, the thermal expansion deforms more and more the substrate. Furthermore, by fitting the curves with the same function described in 3.1, we observe that the linear growth rate of both the horizontal and vertical divergences is increasing with the repetition rate, as shown in Fig. 5. However, a clear behavior cannot be extracted from the exponential constant.

By combining the two previous scans, we can study the change of divergence depending on the average power into the compressor which is varied by changing the input energy and the repetition rate. Fig. 6 shows the average divergence of the horizontal and vertical planes after 30 minutes for all different average power scanned. We further distinguish the data sets acquired before or after the coating deterioration. Nevertheless, the data is in good agreement with the fitted linear slope, which tends to validate the use of continuous diode lasers to reproduce the 

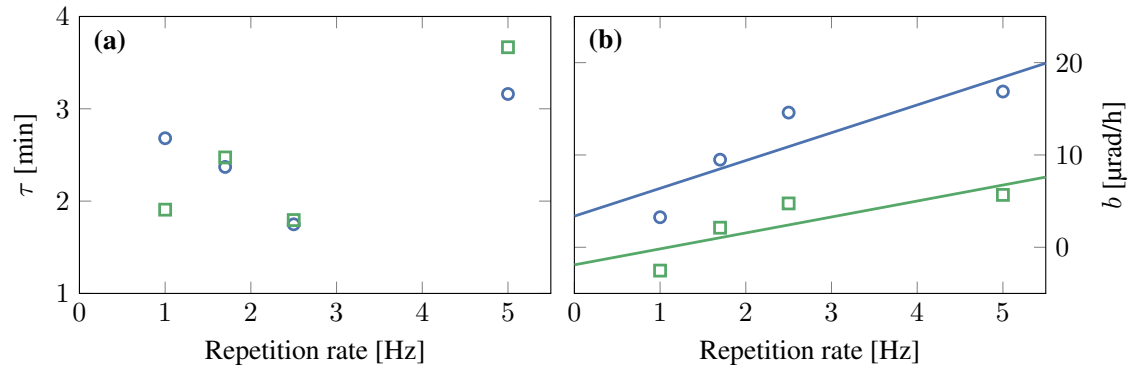

Fig. 5. Exponential constant (a) and linear growth rate (b) of the fitted curves (solid lines on Fig. 4) for the different repetition rates in the horizontal (blue circles) and vertical (green squares) planes. A linear fit (solid line) highlights the behavior of the scan, as previously shown.

behavior observed with a compressed pulse [12]. However, we do observe with the thermal camera that the first grating temperature is significantly higher at the output beam height where the beam is compressed, even with a good coating quality. It would indicate that more energy is absorbed in the substrate where the laser pulse is short, even though the pulse energy is already decreased by at least $85 \%$ due to the grating efficiency of the first three passes.

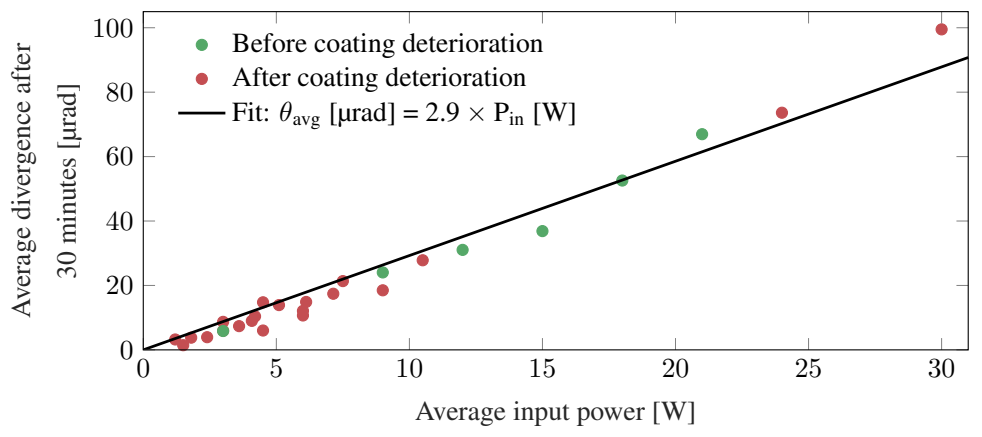

Fig. 6. Average divergence measured after 30 minutes in function of the laser input average power, ranging from $1.2 \mathrm{~W}$ to $30 \mathrm{~W}$. The data (dots) agrees well with the linear fit (solid line), which equation is given in the legend.

\section{Effects on the focal spot quality}

We observed that the heat-induced deformation of the gratings leads to an important increase of the divergence and astigmatism due to the asymmetry in the vertical and horizontal planes. Therefore, it appears necessary to investigate the evolution of the focal spot quality.

Using the wavefront sensor, we monitor the evolution of the relative rms amplitude of the wavefront as well as the relative Strehl ratio, calculated from the point spread function (PSF) using the measured wavefront map. The tilts and the defocus are excluded from the analysis in order to restrict the observations to the quality of the focal spot rather than its longitudinal or transverse position. While the relative Strehl ratio does not indicate the quality of the laser beam directly, it captures the change of its focusability due to the deformation of the grating surface. Figure 7 shows these two measurements for three different input fluences at $5 \mathrm{~Hz}$. At $10 \mathrm{~mJ} / \mathrm{cm}^{2}$, the wavefront and the Strehl ratio stay almost constant to an average value of $\lambda / 20$ and 0.9 respectively. Over 30 minutes, the wavefront amplitude increases by $0.01 \lambda$ and the 

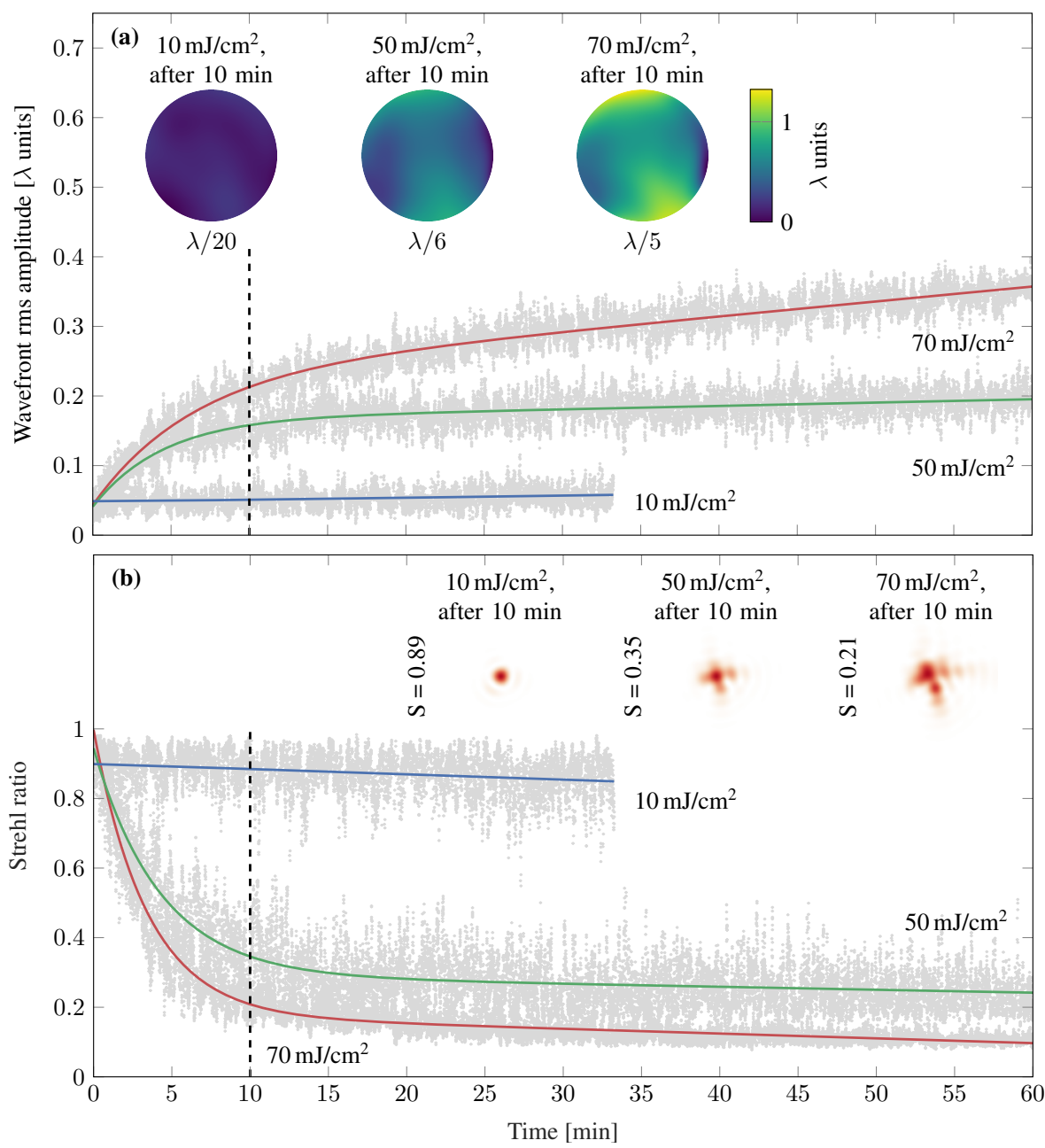

Fig. 7. Evolution of the wavefront rms amplitude (a) and the Strehl ratio of the point spread function (b) for fluences of $10 \mathrm{~mJ} / \mathrm{cm}^{2}$ (blue), $50 \mathrm{~mJ} / \mathrm{cm}^{2}$ (green) and $70 \mathrm{~mJ} / \mathrm{cm}^{2}$ (red) into the compressor at $5 \mathrm{~Hz}$, which corresponds to average power of $3 \mathrm{~W}, 15 \mathrm{~W}$, and $21 \mathrm{~W}$. The data (gray dots) is fitted similarly to the divergence (solid color line). The insets show respectively the wavefront map and the normalized PSF spatial profile for the three fluence levels after 10 minutes with the corresponding wavefront rms amplitude and Strehl ratio value.

Strehl ratio decreases by 0.05 . At $50 \mathrm{~mJ} / \mathrm{cm}^{2}$ and $70 \mathrm{~mJ} / \mathrm{cm}^{2}$, we observe a behavior similar to the divergence evolution: after an exponential growth of the wavefront deformations with a time constant of about 5 minutes, they increase linearly. Especially for the $70 \mathrm{~mJ} / \mathrm{cm}^{2}$ case, which is close to the designed operating parameters of the compressor, the Strehl ratio is reduced to 0.5 in 3 minutes and to 0.2 after 10 minutes. This fast degradation of the laser beam spatial quality reduces heavily its usability as a driver for experiments relying on parameter scans, when operating at high average power.

In order to observe the evolution of the pulse temporal width, we monitored the width of a single-shot second order autocorrelation trace at the post-compressor diagnostics (not shown in Fig. 1). We could observe a broadening of the second harmonic trace as the deformation of the grating surface increased. In principle, spatio-temporal couplings from the deformed gratings 
could cause a change in the compressed laser pulse length [8]. However, due to the complexity of the measured signal, we cannot conclude that this observed broadening is solely related to an increase of the pulse duration. Indeed, as the autocorrelator is not imaging a plane within the compressor, a change of the laser wavefront would additionally modify the near field distribution and therefore the second harmonic signal. We plan to investigate the evolution of the pulse duration in the future using a single-shot device such as a FROG, which can measure some spatio-temporal coupling [16], and reducing the influence of the wavefront degradation on the intensity distribution by imaging a plane in the compressor. However, this is beyond the scope of the current study.

Combining all fluence and repetition rate scans as previously, we use the Strehl ratio value calculated after 30 minutes as a metric in order to estimate an average power threshold above which the wavefront is too degraded to use the output compressed laser beam for experiments. In the following, we will distinguish whether the coating was deteriorated or not. The data represented in Fig. 8 features two regions with rather constant Strehl ratio: at low input power (typically below $5 \mathrm{~W}$ ), the deformations of the gratings are negligible and the Strehl ratio stays above 0.9. On the other hand at high average power (above $15 \mathrm{~W}$ ), the PSF is already so degraded that an additional deformation of the gratings surface will not change significantly the Strehl ratio. From these two observation, the data is fitted by a high order Gaussian curve $\propto \exp \left(\ln \left(S_{\lim }\right) \cdot\left(P / P_{\lim }\right)^{2 N}\right)$, to obtain two plateau-like regions at low and high average power, with a smooth transition in-between. From this fit, the order of the Gaussian is $N=1.2$ and we can retrieve the average power limit for a given Strehl ratio value $S_{\mathrm{lim}}$. For instance, at $S_{\mathrm{lim}}=0.9, P_{\mathrm{lim}}=5.6 \mathrm{~W}$ and at $S_{\text {lim }}=0.8, P_{\text {lim }}=7.7 \mathrm{~W}$. We emphasize that this curve is only a tool to extract a threshold value from the data, and that it depends on the specific compressor design and thermal properties of the setup (e.g. grating mounts).

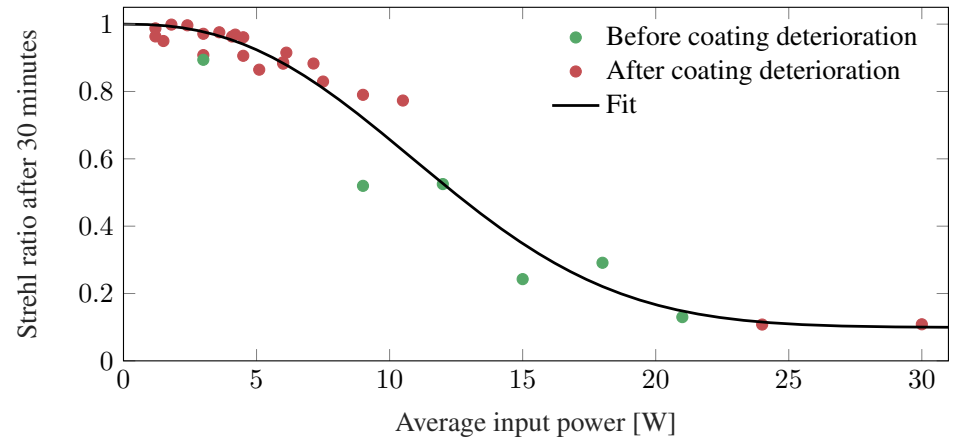

Fig. 8. Strehl ratio calculated from the PSF after 30 minutes in function of the laser input average power, ranging from $1.2 \mathrm{~W}$ to $30 \mathrm{~W}$. The data (dots) is fitted by a high order Gaussian curve (solid line).

Seeing how fast the wavefront degrades due to the gratings surface deformation, it is also of interest to investigate how long the gratings need to recover their initial shape. Thus, after a 90 minutes run at a fluence of $80 \mathrm{~mJ} / \mathrm{cm}^{2}$ at $5 \mathrm{~Hz}$, which corresponds to an average power of $24 \mathrm{~W}$, the laser energy was decreased to $150 \mathrm{~mJ}$ (or $0.75 \mathrm{~W}$ ) in order to significantly reduce the absorbed power but still be able to measure the wavefront deformations, and recorded for 30 minutes, as shown in Fig. 9. After 15 minutes, the Strehl ratio reaches the 0.5 level, and according to the exponential plus linear fit, it would reach the 0.9 level after 50 minutes as the linear slope corresponds to an improvement of $80 \% / \mathrm{h}$.

In principle, a mitigation of the wavefront degradation using a deformable mirror might be feasible. However, as the wavefront changes significantly within only minutes after the laser 

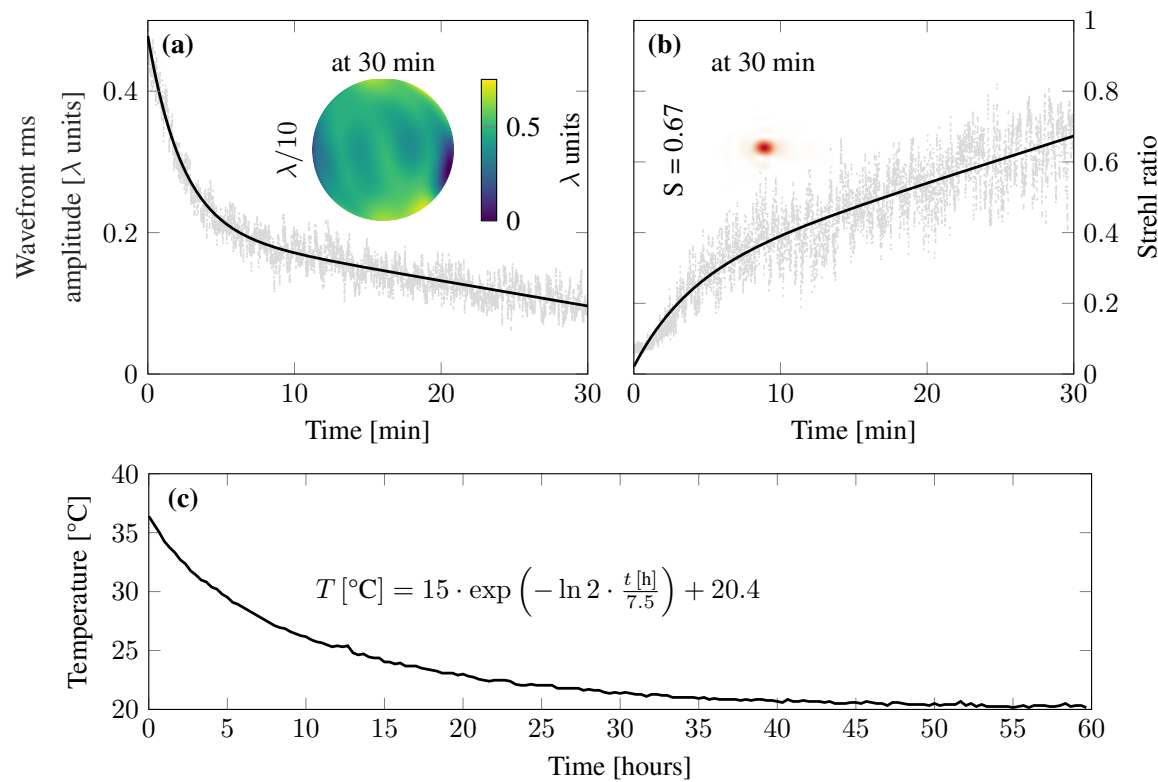

Fig. 9. Evolution of the wavefront rms amplitude (a) and the Strehl ratio of the point spread function (b) after a 90 minutes run at $80 \mathrm{~mJ} / \mathrm{cm}^{2}$ at $5 \mathrm{~Hz}$. The insets show respectively the wavefront and the point spread function profile after 30 minutes. The substrate temperature after a 5 hours run at a $30 \mathrm{~W}$ average power is also reported (c).

energy is reduced, such a correction has likely to be performed online while running at full power. Furthermore, an online adaptive optics loop has to ensure that no intermediate foci are created at full power while the deformable mirror moves the different actuators.

As we were not able to directly measure the temperature of our Pyrex substrates during the experimental campaign, we recorded in a similar experiment the temperature of a fused silica substrate grating, which has a comparable thermal conductivity in the order of $1 \mathrm{~W} /(\mathrm{m} \mathrm{K})$. Therefore, we expect the Pyrex gratings to exhibit a similar thermal behavior as the fused silica substrate grating. The temperature of the fused silica grating was measured for several days after a 5 hours run with an input fluence above $90 \mathrm{~mJ} / \mathrm{cm}^{2}$ at $5 \mathrm{~Hz}$ using a thermal camera. The maximum temperature observable on the side of the grating is reported in Fig. 9, and follows an exponential decay with a half-life of 7.5 hours. We note that during the heating process, the temperature increased linearly with a rate of $3{ }^{\circ} \mathrm{C} / \mathrm{h}$.

\section{Conclusion}

We investigated the divergence change and wavefront degradation of a high repetition rate $100 \mathrm{TW}$ class laser due to laser induced heat in the final compressor gratings with Pyrex substrate, measured directly with the laser used for experiments. Pyrex substrates are widely used in compressors for this class of laser systems, and we therefore believe that our findings are highly relevant for the community. The data shows a significant increase of the divergence and a degradation of the transmitted wavefront if the input power is higher than $5.6 \mathrm{~W}$. We noticed an asymmetric behavior of the divergence between the horizontal and vertical planes which require more detailed analysis and simulations to be better understood. The long-term analysis indicates, that operation of the laser at full average power requires an online adaptive optics correction loop of the transient wavefront deformations. Indeed, the focal spot quality and longitudinal position would otherwise vary during an experiment. It would therefore reduce the effectiveness of 
parameter scans to isolate individual causes of the studied phenomenon. Moreover, we observed a broadening of a second order autocorrelation trace, but we cannot conclude that it is directly related to an increase of the compressed pulse duration. More complete measurements are necessary to investigate the effects of the gratings deformation on the pulse temporal profile. Finally, we observed that the first grating substrate temperature is higher around the compressed pulse which tends to suggest that the ultrashort nature of the beam needs to be considered for an accurate simulation of the heating process. Several options are foreseen to circumvent this issue: actively cooling the grating, as reported in [12], would help to extract the heat stored in the substrate, which reduces the overall surface deformation and helps to reach a thermal equilibrium, and use a grating substrate with a lower thermal expansion coefficient than Pyrex, such as a low expansion glass which have a typical thermal expansion coefficient on the order of $10^{-8} \mathrm{~K}^{-1}$, compared to $\alpha_{\text {Pyrex }}=3.25 \times 10^{-6} \mathrm{~K}^{-1}$. While it would not reduce the temperature increase of the substrate, it would reduce greatly the deformation of the surface and therefore the induced wavefront aberrations. Considering practical use cases of a typical few-hours operation for parameter scans, this might be the most straightforward solution: preliminary results using a fused silica substrate $\left(\alpha_{\text {fused silica }}=0.5 \times 10^{-6} \mathrm{~K}^{-1}\right.$ ) for the first grating already show a clear reduction of the wavefront degradation. However, for a accurate comparison, both gratings need to be replaced. We will therefore report our findings on different grating substrates in the future.

\section{Funding}

Deutsches Elektronen-Synchrotron (DESY); Universität Hamburg; Bundesministerium für Bildung und Forschung (BMBF) (05K16GU2); European Regional Development Fund (CZ.02.1.01/0.0/0.0/16_019/0000789).

\section{Acknowledgments}

The authors acknowledge the equipment contributed by ELI Beamlines. 